[95] The biologic basis of conflict in behaviour.-G. E. Coghill. Psychoanalytic Review, 1933, 20, 1.

THE purpose of this paper is to show that there are, in the development of behaviour, two essentially antagonistic principles, and that these principles prevail also in the development of the nervous system. It is noted first, that the early behaviour pattern of the individual expands as a primarily integrated unit; secondly, that partial patterns arise by individuation within the integrated total pattern; thirdly, that there is a definite neural mechanism of the total pattern; fourthly, that mechanisms of partial patterns arise secondarily and are essentially antagonistic to the mechanism of the total pattern ; fifthly, that the mechanism of the total pattern is a growing organ. Finally, the suggestion is offered that these antagonistic processes may be the organic basis of psychic conflict.

C. S. R.

[96] What is the psychology of organic epilepsy?-L. Pierce Clark. Psychoanalytic Review, 1933, 20, 79.

THE brain and the body are essential in the ego's task of meeting reality; and where these are injured in any way there is a severe handicap to the personality as a whole. An organic lesion may not only cripple the brain but commonly puts out of action certain parts of the body which are also useful to the ego in carrying out its ordinary functions. The injury is a wound to the ego's sense of well-being and completeness; some form of emotional compensation is necessary. There is the tendency for the ego to impound more libido into the resulting narcissism than is really necessary. Such overcompensation has two harmful results. Firstly, it tends to cramp the individual in the full development of his emotional energy towards adult capacities, and there may be narcissistic fixation. Secondly, because of the failure to discharge libido objectively, there is a steady inbinding of tension within the ego. Epileptic attacks, perhaps originally traceable to the lesion itself, now become exaggerated as a mandatory, automatic discharge of this tension. Moreover, the ego in its weakness is characteristically unable to sustain an aggressive socialized use of the destructive impulses, and these, too, are mainly turned inward. Again the impulsive energy is directed upon the ego-in this instance with a violence which is expended in the convulsion. Much may be done in helping the ego to gain strength and to avoid the excessive inbinding of narcissistic libido.

C. S. R.

\title{
NEUROSES AND PSYCHONEUROSES
}

[97] Masked thyrotoxicosis simulating primary neurosis.-S. Ginsburg. Jour. Nerv. Ment. Dis., 1932, 76, 331.

A STUDy of six cases and a review of the literature lead the author to conclude that, 
Thyrotoxicosis is a disease which affects persons of all ages from early infancy to advanced life.

Emotional shock and strain-biological, social-economic, or sexualtraumatic - in apparently normal or neuropathic individuals, may lead to the clinical development of exacerbation of atypical thyrotoxicosis.

Marked thyrotoxicosis may exist for many years without any thyroid enlargement or exophthalmos.

The constitutional symptoms of thyrotoxicosis resemble closely the manifestations of the neuroses and, in the absence of thyroid enlargement and exophthalmos, have been mistaken for pure neuroses.

Many cases of nervous indigestion have been proved to be thyrogenous in origin.

A depressive psychosis may develop in the course of thyrotoxicosis, not as a mere casual phenomenon, but definitely dependent upon toxic thyroid products. Such a psychosis has cleared up and failed to recur when the thyrotoxic condition was controlled.

In cases of neurosis or depressive psychosis associated with tachycardia, tremor, heat intolerance, excessive sweating, loss in weight, diarrhœa with or without abdominal cramps, with or without thyroid enlargement-the possibility of thyrotoxicosis should be thought of and careful studies made to establish or rule out its presence.

The most valuable single corroborative laboratory test when positive is the presence of an increased basal metabolic rate. However, in atypical or borderline cases of thyrotoxicosis the basal metabolic rate may be only slightly raised or normal.

In doubtful cases the iodin therapeutic-diagnostic test, with marked amelioration or aggravation of symptoms after iodin medication, may be helpful in determining the presence of thyrotoxicosis.

Similarly, the radium therapeutic-diagnostic test helps in atypical or borderline cases to differentiate between thyrotoxicosis and pure neurosis. Marked clinical improvement following radium application over the thyroid speaks in favour of an underlying thyrotoxicosis.

The radium therapeutic-diagnostic test properly carried out by an experienced radium therapist is a safe and dependable method, yielding the desired information without subjecting the patient to the dangers of operation and its frequent sequelæ, e.g. myxodema.

R. G. G.

\section{PSYCHOSES}

[98] Crime in schizophrenic types.-James Hunter Murdoch. Jour. of Ment. Sci., 1933, 79, 286.

From his observations on criminal statistics, the author notes that crime in schizophrenics is found most frequently at those times when crimes are most 